\title{
Síndrome de Burnout em enfermeiros atuantes em unidade de terapia intensiva
}

Recebido em: 25/09/2013

Aprovado em: 14/08/2014.
Glébia Keila da Cunha Silva'

Fernanda Valéria Silva Dantas Avelino ${ }^{2}$

Márcia Teles de Oliveira Gouveia ${ }^{3}$

Maria Zélia De Araujo Madeira ${ }^{4}$

Francinete Paula Dantas Avelino ${ }^{5}$

Resumo: Estudo descritivo-exploratório, transversal, com amostra de 33 enfermeiros, objetivou verificar a ocorrência da síndrome de burnout em enfermeiros atuantes em unidade de terapia intensiva. Foram aplicados questionários dos dados sociodemográficos e o Maslach Burnout Inventory. Dos participantes, $43 \%$ apresentaram baixo nível de exaustão emocional, $52 \%$ baixo nível de despersonalização, 49\% demonstraram satisfação moderada no trabalho e em $6 \%$ foi diagnosticado o burnout. Concluiu-se que a ocorrência da síndrome nos enfermeiros é sutil, pois para diagnosticá-la deve-se obter nível alto para exaustão emocional e despersonalização, e nível baixo para realização profissional.

Descritores: Síndrome de burnout, Unidade de terapia intensiva, Enfermagem.

\section{Burnout syndrome in nurses working in intensive care unit}

Abstract: . A descriptive and exploratory study, cross-sectional with the sample of 33 nurses with the objective to determine the occurrence of burnout in nurses working in intensive care unit. We used the questionnaires of sociodemographic data and the Maslach Burnout Inventory. Among the participants, $43 \%$ of nurses showed low level of emotional exhaustion, $52 \%$ low level of depersonalization, $49 \%$ showed moderate satisfaction at work and $6 \%$ were diagnosed with burnout. We concluded that the occurrence of burnout in nurses is subtle, because to diagnose it should get high to emotional exhaustion and depersonalization, and low for professional achievement.

Descriptors: Burnout syndrome, Intensive care unit, Nursing.

\section{Síndrome de Burnout en enfermeros que trabajan en la unidad de cuidados intensivos}

Resumen: Estudio descriptivo y exploratorio, transversal muestra de 33 enfermeros, con el fin de verificar la ocurrencia de burnout en enfermeras que trabajan en la unidad de cuidados intensivos. Los cuestionarios se utilizan para los datos demográficos y el Inventario Burnout de Maslach. Entre los participantes, el $43 \%$ de las enfermeras tenían bajos niveles de agotamiento emocional, $52 \%$ bajo nivel de despersonalización, el $49 \%$ mostró una moderada satisfacción en el trabajo y en el $6 \%$ fue diagnosticado agotamiento. Se concluyó que la aparición del síndrome en enfermeros es sutil, porque el diagnóstico se debe drogarse hasta el agotamiento emocional despersonalización,y el bajo nivel de logro profesional

Descriptores: Síndrome de Burnout, Unidad de Cuidados Intensivos, Enfermería.

\section{INTRODUÇÃO}

$\mathrm{O}$ desenvolvimento tecnológico, sócio-cultural e as conseqüências da globalização econômica geram benefícios ao mundo moderno e, em contrapartida, trazem profundas mudanças no comportamento biopsicossocial do ser humano. As profissões humanas exigem do trabalhador cada vez mais responsabilidades e novos conhecimentos tecnológicos, adequados à ambientes de trabalhos também modificados ${ }^{(1)}$.

A organização do trabalho exerce sobre o homem um impacto no aparelho psíquico que, em certas condições, emergem sofrimentos relacionados a sua história individual. Essas exigências podem superar o limite do homem, induzindo-o ao estresse. E os desgastes a que as pessoas podem obter no ambiente e relações de trabalho implicam em fator categórico de doenças ${ }^{(2,3)}$.

A síndrome de burnout vai além do estresse, sendo encarada como uma reação ao estresse crônico ${ }^{(4)}$.

A síndrome de Burnout começou a ser divulgada de forma inédita a partir de 1974 com o psicanalista Herbert J. Freudenberger, que a apresentou como um sentimento negativo, de fracasso e de exaustão conseqüentes do excessivo desgaste de energia e de recursos ${ }^{(5)}$.

Após Freudenberger, Christina Maslach foi outra precursora dos estudos referentes à síndrome de burnout em profissionais que trabalham intensamente com recursos humanos como: médicos, enfermeiros, advogados, professores dentre outros. Assim, Freudenberger e Christina Maslach são reconhecidos como os teóricos mais importantes no que diz respeito à síndrome de burnout ${ }^{(6)}$.

No Brasil, foi mencionada pela primeira vez em 1987, sendo posteriormente incluída na lista de doenças relacionadas ao trabalho na Classificação Internacional das Doenças (CID-10) no Grupo V (Transtornos Mentais e do Comportamento Relacionado ao Trabalho), com o código $Z 73^{(7),(5)}$.

Definida como uma das conseqüências mais acentuadas do estresse profissional, a síndrome de burnout apresenta três 
dimensões sintomatológicas: (1) exaustão emocional, em que o individuo revela inicialmente um forte abalo físico, psíquico e emocional; (2) a despersonalização que mostra um profissional frio e insensível ao sofrimento do próximo e (3) a baixa realização profissional manifestando-se como uma insatisfação e desânimo no que faz, comprometendo assim a eficiência, a habilidade de realizar tarefas e a adequação à organização do trabalho ${ }^{(8),(9)}$.

Os sintomas da síndrome de Burnout podem ser expostos em quatro grupos: físicos (fadiga constante e progressiva, distúrbios do sono, dores osteomusculares, perturbações gastrointestinais, transtornos cardiovasculares, distúrbios do sistema respiratório, disfunções sexuais), psíquicos (falta de atenção e concentração, alterações de memória, impaciência, sentimento de insuficiência, baixa auto-estima, labilidade emocional, depressão), comportamentais (negligência ou excesso de escrúpulos, irritabilidade, incremento da agressividade, incapacidade de relaxar, perda da iniciativa, comportamento de alto risco, suicídio, desconfiança e paranóia) e defensivos (tendência ao isolamento, sentimento de onipotência, perda do interesse pelo trabalho, absenteísmo, ironia, cinismo) ${ }^{(10),(6)}$.

As conseqüências da síndrome são severas e em muitos casos irreversíveis. Em alto nível o Burnout faz com que os profissionais desejem um curto tempo de trabalho, longo período de férias ou até mesmo a demissão ${ }^{(11)}$.

Entre os fatores vinculados ao desenvolvimento da síndrome de Burnout estão: necessidade de reconhecimento, subvalorização profissional, necessidade de superação profissional, sobrecarga de trabalho; necessidade de trabalho em equipe, necessidade de autonomia e características da tarefa ou rotina de trabalho(2).

Relacionada com a exposição prolongada aos elementos facilitadores do estresse laboral e a ausência de apoio social ocasionando danos físicos e psíquicos ao trabalhador, a síndrome de Burnout atinge distintas categorias profissionais, principalmente os que requerem maior contato interpessoal. Conforme o Health Education Authority a enfermagem é classificada em quarto lugar como a profissão mais estressante em âmbito público ${ }^{(12),(13)}$.

Destaca-se que o estresse possui elevada correlação com o termo burnout, entretanto enquanto o primeiro possui aspectos positivos e negativos, o segundo restringe-se aos aspectos negativos, sendo de caráter crônico, inadaptativo desencadeado lentamente de modo progressivo e cumulativo. Tais semelhanças dificultam o diagnóstico preciso, implicando em tratamentos inadequados e na piora do quadro(3).

O estudo da síndrome de Burnout é de grande relevância para a saúde do trabalhador visto que pode causar modificações significativas nos profissionais comprometendo sua vida pessoal e seu trabalho. Em razão das altas demandas físicas e emocionais associadas ao ambiente de trabalho, as enfermeiras representam grupo ocupacional especialmente exposto a sofrer a síndrome de Burnout ${ }^{(14)}$.

Desta forma este estudo objetiva verificar a ocorrência da síndrome de burnout em enfermeiros que prestam assistência a pacientes internados nas unidades de terapias intensivas (UTI) dos hospitais de ensino da cidade de Teresina, Piauí.

\section{MÉTODOLOGIA}

Trata-se de um estudo descritivo-exploratório com abordagem quantitativa do tipo corte transversal, realizado em Unidades de Terapia Intensiva (UTI) de quatro hospitais de ensino da cidade de Teresina, referências na prestação de serviços de alta e média complexidade em saúde.
A população do estudo consistiu de 40 enfermeiros que exerciam ativamente suas funções nos locais de pesquisa; perfazendo uma amostra final de 33 profissionais que assinaram o Termo de Consentimento Livre e Esclarecido. Por envolver seres humanos, foi necessária a avaliação ética deste trabalho, em cumprimento às determinações éticas previstas conforme a Resolução no. 196/96, que adota como diretrizes os princípios fundamentais da bioética: autonomia, beneficência, não-maleficência e justiça ${ }^{(15)}$.

Entre os critérios de inclusão destacam-se: disponibilidade para participar deste estudo, ser enfermeiro e estar exercendo ativamente suas funções nos locais de estudo com tempo mínimo de 01 (um) ano de atuação em UTI.

Os dados foram coletados, logo após a aprovação pelo Comitê de Ética e Pesquisa da Universidade Federal do Piauí (CEP-UFPI), CAAE nº 0408004500010 e das quatro instituições hospitalares onde foi executada a pesquisa, ocorrendo durante os meses de março a maio de 2011.

Utilizou-se o questionário proposto por Benevides-Pereira (2002), que armazenou dados demográficos e profissionais e os adicionou ao instrumento de Maslach Burnout Inventory (MBI), composto por 22 questões. A síndrome de burnout segundo o manual do $\mathrm{MBI}$ é diagnosticada no individuo por meio da obtenção de nível alto para exaustão emocional e despersonalização e nível baixo para realização profissional. Sendo assim, o ajuste do profissional nesses três aspectos define a manifestação da doença. E o somatório das três dimensões resulta na classificação dos níveis da síndrome de Burnout. ${ }^{(10,16)}$

Os dados dos questionários foram processados através do programa SPSS 16.0 (Statistical Package for the Social Sciences).

A pontuação dos itens pesquisados em relação ao questionário do $\mathrm{MBI}$ adotou uma escala do tipo Likert que varia de 0 a 6 pontos, sendo: (0) nunca, (1) uma vez ao ano ou menos, (2) uma vez ao mês ou menos, (3) algumas vezes no mês, (4) uma vez por semana, (5) algumas vezes por semana, (6) todos os dias. Os valores do somatório obtidos pelo $\mathrm{MBI}$ foram comparados com os da referência do Núcleo de Estudos Avançados sobre a Síndrome de Burnout (NEPASB) e obteve-se conseqüentemente a classificação do nível da síndrome.

\begin{tabular}{|ccccc|}
\hline Dimensões & Questões & \multicolumn{3}{c|}{ Padrão de Pontuação } \\
\hline $\begin{array}{c}\text { Exaustão } \\
\text { emocional }\end{array}$ & 1 a 9 & 26 & $16-25$ & 15 \\
Rével alto & $\begin{array}{c}\text { Nível } \\
\text { médio }\end{array}$ & Nível baixo \\
Realização pessoal & 10 à 17 & 43 & $34-42$ & 33 \\
\hline Despersonalização & 18 à 22 & 9 & $3-8$ & 2 \\
\hline
\end{tabular}

Fonte $^{10}$.

Quadro 1. Padrão de pontuação para diagnóstico das dimensões da síndrome de Burnout pelo Maslach Burnout Inventory (MBI), 2008. 
TABELA 1 - Distribuição da amostra segundo características sócio-demográficas. Teresina (PI), 2011.

\begin{tabular}{|c|c|c|c|}
\hline \multicolumn{2}{|c|}{ Variável } & $\mathbf{N}$ & $\%$ \\
\hline \multirow{2}{*}{ Sexo } & Feminino & 31 & 94 \\
\hline & Masculino & 2 & 6 \\
\hline \multirow{2}{*}{ Faixa Etária } & $22-40$ & 21 & 64 \\
\hline & $41-62$ & 12 & 36 \\
\hline \multirow{3}{*}{ Estado Civil } & Solteiro & 12 & 36 \\
\hline & Casado & 17 & 52 \\
\hline & $\begin{array}{l}\text { Divorciado/ } \\
\text { Separado }\end{array}$ & 4 & 12 \\
\hline \multirow{2}{*}{ Filhos } & Sim & 23 & 70 \\
\hline & Não & 10 & 30 \\
\hline \multirow{3}{*}{$\begin{array}{c}\text { Vínculo } \\
\text { Empregatício }\end{array}$} & Estatutário & 24 & 73 \\
\hline & Temporário & 3 & 9 \\
\hline & $\begin{array}{c}\text { Carteira } \\
\text { assinada/Outro }\end{array}$ & 6 & 18 \\
\hline \multirow{2}{*}{$\begin{array}{c}\text { Carga Horária } \\
\text { Semanal }\end{array}$} & $20-40$ & 29 & 88 \\
\hline & $44-66$ & 4 & 12 \\
\hline \multirow{2}{*}{ Atividade Física } & Sim & 18 & 55 \\
\hline & Não & 15 & 45 \\
\hline Total & & 33 & 100 \\
\hline
\end{tabular}

Fonte: Pesquisa direta.

Constatou-se a prevalência do sexo feminino (94\%), com idades entre 22 a 40 anos (64\%) e 41 a 62 anos (36\%). Essa proporção em relação ao sexo era esperada, pelo fato da própria categoria profissional de enfermagem ser composta, sobretudo por mulheres ${ }^{(17)}$. Quanto à idade, estudos relacionam que o aumento da faixa etária em anos é um fator que contribui para a redução da manifestação da síndrome de Burnout por acreditarem que os profissionais possuem certa maturidade alcançada pelas experiências e qualidade no trabalho(9), (8), (18).

Em relação ao estado civil predominou a porcentagem de casados (52\%), seguidos pelos solteiros (36\%) e $70 \%$ da amostra relataram ter filhos. A existência de relação matrimonial e filhos possui uma menor correlação ao surgimento da síndrome de Burnout ${ }^{(5),(9),(16)}$.

Quanto ao vínculo empregatício 73\% afirmaram ser estatutários. Dos profissionais pesquisados nota-se que a maioria (88\%) possuía carga horária de trabalho entre 20 a 40 horas semanais. O excesso de horas de trabalho é apontado como um dos fatores responsáveis pela manifestação dos sintomas da síndrome de Burnout ${ }^{(13)}$.

Na tabela 1 observou-se que, 55\% dos enfermeiros praticavam algum tipo de atividade física. A respeito da atividade física a literatura reconhece que os exercícios auxiliam na redução de tensões diminuindo o estresse e atuando na manutenção da condição de saúde no trabalho(10),(16).

Através da aplicação do instrumento de Maslach Burnout Inventory (MBI) foi possível detectar a ocorrência da síndrome de Burnout em 2 enfermeiros, correspondendo a um percentual de $6 \%$.

A Tabela 2 mostra o nível das dimensões da síndrome de Burnout em enfermeiros atuantes nas unidades de terapia intensiva.
TABELA 2 - Distribuição por nível das dimensões da síndrome de Burnout conforme MBI. Teresina (PI), 2011.

\begin{tabular}{cccccccc} 
Nível & \multicolumn{2}{c}{$\begin{array}{c}\text { Exaustão } \\
\text { Emocional }\end{array}$} & \multicolumn{2}{c}{ Despersonalização } & \multicolumn{2}{c}{$\begin{array}{c}\text { Realização } \\
\text { profissional }\end{array}$} \\
Alto & $\mathbf{N}$ & $\%$ & $\mathbf{N}$ & $\%$ & $\mathbf{N}$ & $\%$ \\
Médio & 10 & 27 & 5 & 13 & 11 & 33 \\
Baixo & 14 & 43 & 11 & 33 & 16 & 49 \\
\hline
\end{tabular}

Fonte: pesquisa direta.

Verificou-se que, em consonância com a tabela 2, do grupo de 33 enfermeiros pesquisados: a exaustão emocional apresentou nível médio a baixo (73\%), a despersonalização prevaleceu em nível médio a baixo (85\%), enquanto a realização profissional apresentou nível médio a alto (82\%).

Esses dados mostram que a maioria dos enfermeiros, possui baixo e médio nível para exaustão emocional, em concordância com um estudo realizado em uma equipe de resgate pré-hospitalar do município de São Paulo que apontou $76,47 \%$ dos profissionais com baixo e médio nível para essa dimensão de Burnout ${ }^{19}$.

Quanto à despersonalização nota-se que a maioria dos enfermeiros apresentou nível baixo (52\%), resultado compatível com estudo envolvendo enfermeiros no Rio Grande do Norte, que apresentaram $66 \%$ profissionais com baixo nível de despersonalização ${ }^{20}$.

Acerca da realização profissional, 49\% dos enfermeiros mencionaram estar satisfeitos de forma moderada com seu trabalho e 33\% mostraram alta satisfação. Totalizando $82 \%$ dos profissionais com nível médio e alto de realização profissional. Em um estudo semelhante, 58,62\% dos trabalhadores de enfermagem apresentaram nível alto e $24,13 \%$ nível médio para essa dimensão, obtendo-se uma soma de $82,75 \%$ dos enfermeiros com media e alta realização profissional ${ }^{7}$.

A respeito dos sintomas somáticos decorrentes do trabalho, detectou-se que os mais freqüentes foram: dores nos ombros ou nuca ( $86 \%)$, cefaléia (71\%), pouco tempo para si mesmo (67\%), dificuldade de memória e concentração (57\%), cansaço mental (57\%), irritabilidade fácil, fadiga generalizada e perda de senso de humor (52\%). Os sintomas com maior percentual citados se enquadram nos quatro grupos: físico, psíquico, comportamental e defensivo referidos por Benevides-Pereira (2002) caracterizando a sintomatologia mais freqüente nos profissionais propensos a obtenção da síndrome de Burnout.

Ao correlacionar os dados sociodemográficos dos enfermeiros à presença da síndrome de Burnout, observou-se que ambas eram do sexo feminino, com faixa etária entre 22 a 40 anos, sem filhos, sendo uma casada e a outra solteira. Quanto à atividade física ambas praticavam exercícios. Dessa forma, os resultados exibidos apresentam-se condizentes com a literatura ${ }^{(8),(9),(10),(16),(18)}$.

\section{CONCLUSÃO}

A partir da investigação realizada pode-se concluir que a síndrome de Burnout acontece de forma discreta em enfermeiros de Unidades de Terapia Intensiva nos quatro hospitais de ensino no município de Teresina., Piauí.Entretanto ressalta-se a presença de sintomas somáticos em grande parte dos profissionais; o que se traduz em afecções físicas e psicológicas importantes.

Diante da presença da síndrome de Burnout em enfermeiros de unidades fechadas e complexas, ressalta-se a necessidade 
de estudos voltados para melhor compreensão da doença quanto ao seu significado, sintomatologia e tratamento alem de estimular a atenção dos profissionais da UTI para os fatores de risco aos quais estão expostos no ambiente de trabalho.

Destaca-se que o tratamento da síndrome de Burnout consiste em acompanhamento psicoterápico e farmacológico, associado a intervenções psicossociais. Entretanto faz-se mister a adoção de medidas de prevenção e promoção da saúde ocupacional, como a diversificação de rotinas de trabalho, a prevenção do excesso de horas extras e/ou a sobrecarga de atividades, suporte social aos funcionários, melhores condições sociais e físicas do ambiente de trabalho e investimento no aperfeiçoamento profissional e pessoal dos trabalhadores

\section{Referências}

1 Leitão IMTA, Fernandes AL, Ramos IC. Saúde ocupacional: analisando os riscos relacionados à equipe de enfermagem numa unidade de terapia intensiva. Cienc Cuid Saúde. 2008. 7: 476-84.

2 Veja ML, Pérez IG, Amador YC. Uso el método cualitativo en e estúdio el Burnout. Thompson Psicologia. 2003.1.

3 Magalhães AB. A síndrome de burnout no contexto hospitalar pediátrico [Dissertação]. Goiânia: Universidade Católica de Goiás; 2006.

4 Moraes MVG. Doenças ocupacionais-agentes: físico, químico, biológico, ergonômico. São Paulo: latria, 2010.

5 Campos RG. Burnout: uma revisão integrativa na enfermagem oncológica [Dissertação]. Ribeirão Preto: Escola de Enfermagem de Ribeirão Preto da Universidade de São Paulo; 2005.

6 Lima CF. Síndrome de burnout e autoeficácia: um estudo com profissionais de enfermagem de hospitais privados de Natal/ RN [Dissertação]. Natal: UFRN; 2009.

7 Fascina LP, Guimarães CPA, Hidaka KS, Mekler PL, Rezende F. Avaliação do nível da síndrome de burnout na equipe de enfermagem da UTI adulto. Pesquisa realizada como parte de programação científica, São Paulo, 2007.

8 Moreira DS, Magnago RF, Sakae TM, Magajewski FRL. Prevalência da síndrome de burnout em trabalhadores de enfermagem de um hospital de grande porte na região sul do Brasil. Rio de Janeiro. Cad. Saúde Pública. 2009. 25(7): 1559-68.

\section{Jodas DA, Haddad MCL. Síndrome de burnout em}

trabalhadores de enfermagem de um pronto socorro de um hospital universitário. São Paulo. Acta Paul Enferm. 2009. 22(2):192-7.

10 Benevides-Pereira AMT. Burnout: o processo de adoecer pelo trabalho. In: Benevides-Pereira AMT (Org.) Burnout: quando o trabalho ameaça o bem estar do trabalhador. $3^{a}$ Ed. São Paulo: Casa do Psicólogo. 2008; 21-92.

11 Nobrega-Therrien SM, Almeida MI. Temas em saúde da família: diversidades de saberes e praticas profissionais fundamentadas na pesquisa. Fortaleza: Eduece; 2006.
12 Trindade LL, Lautert L. Síndrome de burnout entre os trabalhadores da estratégia saúde da família. Rev Esc Enferm USP. 2010; 44(2): 274-9.

13 Murofuse NT, Abranches SS, Napoleão AA. Reflexões sobre estresse e burnout e a relação com a enfermagem. Rev Latino-Am Enferm [on line]. [acesso em 15/06/2011]. 2005; 13(2): 255-61. ISSN 0104-1169. http://dx.doi.org/10.1590/S0104-11692005000200019.

14 Diaz-Rodrigues L, Arroyo-Morales M, Cantarero-Villanueva I, Férnandez-Lao C, Polley M, Fernández-des-las-Peñas C. Uma sessão de reiki em enfermeiros com síndrome de burnout tem efeitos benéficos sobre a concentração de $\lg A$ salivar e a pressão arterial. Rev Latino-Am Enferm [online]. [acesso em 10/06/2011]. 2011, 19(5): 1132-8. ISSN 0104-1169.http://dx.doi.org/10.1590/5010411692011000500010.

15 BRASIL. Ministério da Saúde. Secretaria de Assistência à Saúde. Departamento de Normas Técnicas. Manual Operacional para Comitês de Ética em Pesquisa. Brasília-DF: Ministério da Saúde, 2002.

16 Menegaz FDL. Características da incidência de burnout em pediatras de uma organização hospitalar pública [dissertação]. Florianópolis: Centro de Filosofia e Ciências Humanas da Universidade Federal de Santa Catarina; 2004.

17 Gil-Monte PR. Influencia del género sobre el proceso de desarrollo del síndrome quemarse por el trabajo (burnout) en profesionales de enfermería. Psicol Estud. [on line] [acesso em 09/06/2011]. 7(1) 3-10. ISSN 1413-7372. http://dx.doi.org/10.1590/ S1413-73722002000100003.

18 Kebian LVA, Furtado CMSC, Paulino EFR. A síndrome de burnout nos estudos de enfermagem: uma revisão bibliográfica. Revista Corpus et Scientia. Rio de Janeiro. 2010. 6(2): 51-61.

19 Bezerra RP, Beresin R. A síndrome de burnout em enfermeiros da equipe de resgate pré-hospitalar. Einstein. 2009. 7(3): 351-6.

20 Lima CF. Síndrome de burnout e autoeficácia: um estudo com profiisionais de enfermagem de hospitais privados de Natal/ RN.2009.125f. [Dissertação] - Universidade Federal do Rio Grande do Norte, Natal. 2009. 